\title{
Biomechanical comparison of single- double- and novel triple- row rotator cuff repairs in a porcine model using generic anchors
}

\begin{abstract}
Comparison of a novel triple-row (NTR) rotator cuff repair (RCR) was made to single row (SR) and double row (DR) repairs as measured by gap formation, Ultimate Failure Load (UFL), and mode of failure. Porcine humeri were divided into three groups of forty and the infraspinatus tendons were completely released and then repaired using generic (PEEK) anchors. Biomechanical testing of RCRs consisted of 3500 cycles of a load varied from 10 to 180 Newtons (N). Gap size at all cycle counts was significantly larger for SR and DR than NTR RCRs. $84 \%$ of NTR repairs completed the 3500 cycle test versus $66 \%$ of DR and $61 \%$ of SR repairs. The tendon pulled from the sutures $60 \%, 49 \%$ and $33 \%$ of the time for SR, DR and NTR RCRs respectively. UFLs were $464 \mathrm{~N}$ for the NTR repairs, $394 \mathrm{~N}$ for DR and $414 \mathrm{~N}$ for SR repairs. In summary, NTR repairs resulted in smaller gap formation, higher UFLs, and fewer instances of tendons tearing at the sutures than SR or DR repairs. Results of NTR repairs demonstrate that the tendon footprint is securely restored with small gap formation for the range of forces that a shoulder will be subjected to in normal use.
\end{abstract}

Keywords: rotator cuff, rotator cuff repair, shoulder, single row, double row, triple row, porcine, ultimate failure load, biomechanical
Volume 2 Issue 4 - 2015

\author{
Blair A Rhode \\ Orland Park Orthopedics, USA
}

Correspondence: Blair A Rhode, Orland Park Orthopedics, I6450 S 104th AV, Orland Park, Illinois 60467, USA, Tel 708-364844I,Email blairbones@gmail.com

Received: February II, 20I5 | Published: April 29, 2015
Abbreviations: RC,: Rotator Cuff; RCR,: Rotator Cuff Repair; SR, Single Row; DR, Double Row; NTR, Novel Triple Row; UFL, Ultimate Failure Load; N, Newtons; PEEK, Polyetheretherketone; df, Degrees of Freedom; RōG, Rhode Orthopedic Group

\section{Introduction}

Rotator cuff disease is one of the most common shoulder disorders. When surgical intervention is indicated arthroscopic rotator cuff repair (RCR) has become the method of choice. The goal of the repair is to obtain tendon-to-bone fixation with a minimal gap that is maintained while healing proceeds. The optimal RCR method remains controversial. ${ }^{1}$ Biomechanical studies have been performed on both animal models and human cadaver shoulders to determine the relative merits of rotator cuff repair techniques. ${ }^{2-7}$ Comparison of double-row (DR) to single-row (SR) suture-anchor rotator cuff repair have not always resulted in clear advantages of one method over the other. Outcome differences remain in spite of several studies indicate that DR repairs result in better footplate restoration and smaller gap size than SR repairs. ${ }^{8}$ However, when tear size is stratified there is a significant difference between SR and DR repairs. ${ }^{9}$ Clinical studies of DR versus SR repairs often conclude that patient outcomes as measured by gap development do not differ between the two techniques. ${ }^{8}$ Studies often conclude that further research is required to determine whether better footplate restoration in DR repairs results in superior healing as observed by imaging the rotator cuff.

RCR biomechanical studies permit manipulations that cannot be duplicated in patients. Recent studies have employed an increasing number of anchors and sutures in a variety of configurations. ${ }^{10,11}$ The goal of each study was to increase the pressure over the entire footplate of the torn tendon. A triple row repair and a diamondback (DBK) repair employed knotless anchors at the edge of the tendon to allow sutures to compress the tendon edge to the bone. ${ }^{12-14}$ Pressure measurements in the tendon footplate indicated DBK repair was the

\section{best repair of four repair methods.}

Due to the ongoing controversy as to the best repair method and concerns over total cost, a novel triple-row (NTR) repair is compared to DR and SR repairs of porcine infraspinatus tendon tears. NTR repair employs two levels of mattress sutures and two knotless anchors beyond the tendon edge to compress the tendon to its footplate. This approach is reminiscent of the bridging self-reinforcing double-row $\mathrm{RC}$ repair. ${ }^{15}$ Repair performance is determined by Ultimate Failure Loads (UFLs), gap size development, and mode of tendon failure. Clinically RCR involves cost, time, and ease of repair, all of which are minimal in a simple-single row repair. A simple repair doesn't strangulate the tendon and allows little separation from the bone in the absence of significant tension.

Hypothesis: H0: there is no difference between a triple row versus a single or double row RCR as measured by gap size, Ultimate Failure Load (UFL), or mode of tendon failure.

\section{Methods}

120 pig shoulders were obtained from a wholesale meat market and divided into three groups of 40 . All tissue was removed from the humerus except the infraspinatus tendon. The preparation was frozen until used. Porcine infraspinatus tendons are approximately $15 \mathrm{~mm}$ in width and $7 \mathrm{~mm}$ thick in the footplate region. A double layer of sterile wrap (KimgardOे) was sewed to the free end of the tendon using a continuous stitch (Ethicon 0 silk) to prevent the upper grip of the test instrument from compressing the tendon to the point that it results in an early failure at the site of the grip. After the preparation was warmed to room temperature the tendon was released from the humerus with a \#20 scalpel blade. NTR repair employs two generic $5.5 \mathrm{~mm}$ polyetheretherketone (PEEK) anchors (RōG, Orland Park, IL) loaded with two ultrahigh molecular weight polyethylene (UHMWPE) \#2 sutures inserted at a $75-90^{\circ}$ angle to the bone. ${ }^{16}$ The steps in an NTR repair technique are illustrated in (Figures 1-6). 

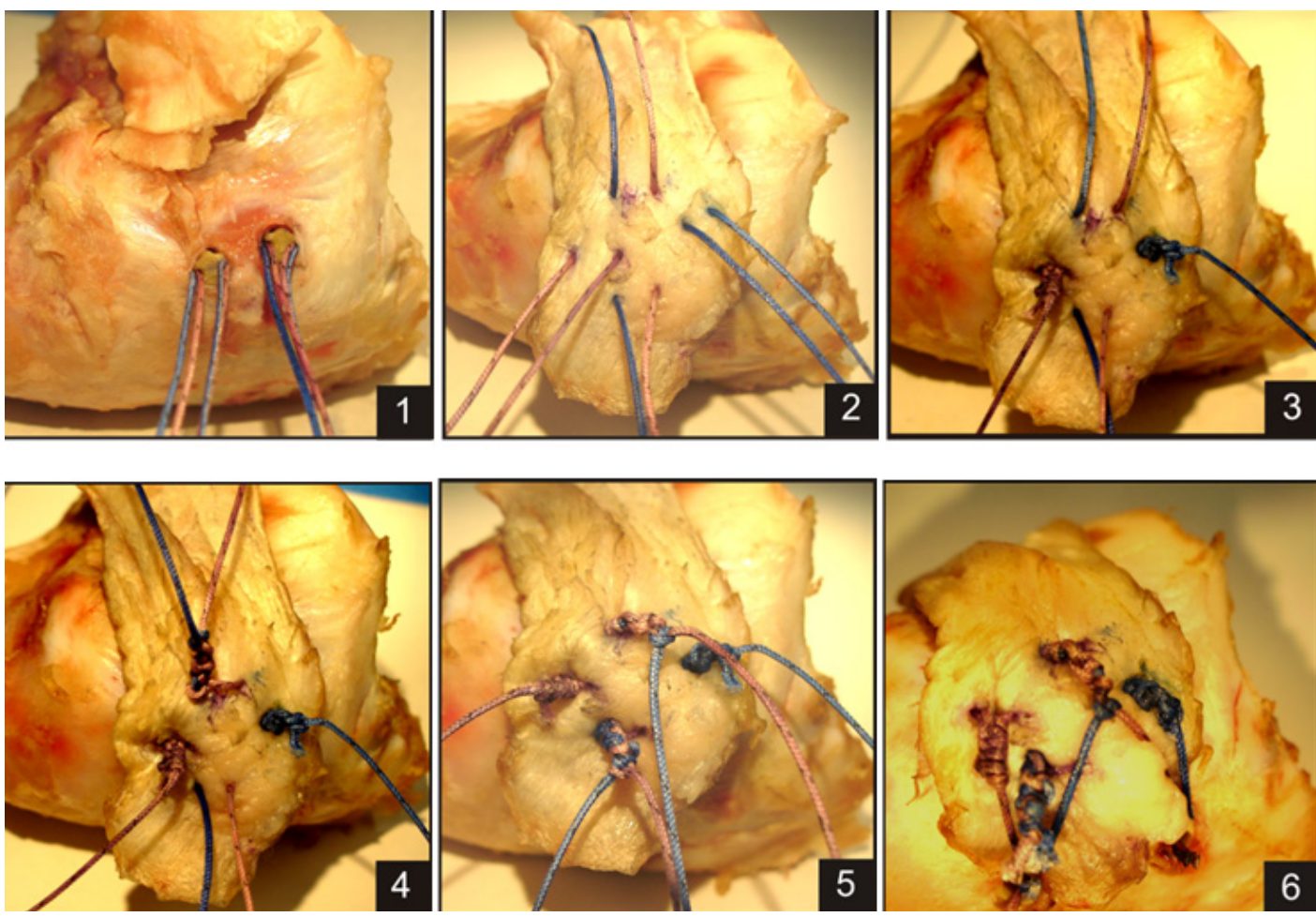

Figure I Step one of the triple row repair begins with inserting two double loaded anchors I cm apart in the tendon footplate.

Figure 2 Position of the four sutures at three levels in the tendon.

Figure 3 Horizontal mattress passes are made through the anterior and posterior margins of the rotator cuff tear.

Figure 4 Medial sutures are tied in air knot fashion.

Figure 5 Medial sutures are reduced onto the medial footprint by traction on the lateral sutures.

Figure 6 Finally, the sutures from the medial rows are passed through the knotless anchors positioned lateral to the footplate and tensioned to hold down the lateral edge of the tendon.
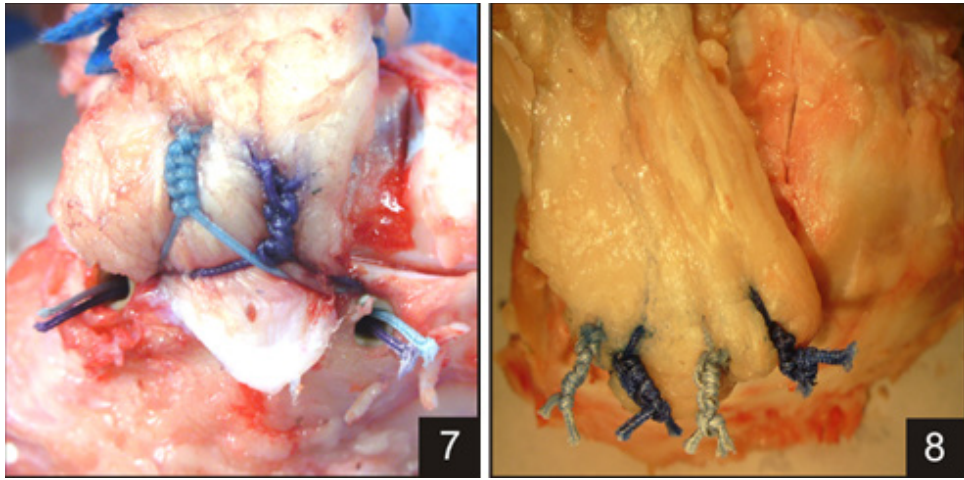

Figure 7 The double row repair employed two $5.5 \mathrm{~mm}$ double-loaded PEEK anchors centered in the tendon footprint separated by I cm with sutures tied in a mattress configuration. Two $5.5 \mathrm{~mm}$ knotless anchors are positioned laterally. Sutures from the medial row are passed through the knotless anchors and tensioned to hold down the lateral edge of the tendon.

Figure 8 Illustration of the single row repair with two double loaded anchors positioned lateral to the tendon footplate. Sutures are passed equally spaced approximately $\mathrm{I} \mathrm{cm}$ from the lateral edge of the tendon.

The humerus was held at a $45^{\circ}$ angle in a special-bilt vise with the tendon at its normal position and angle when the load is applied. Iron shafts are inserted through two holes drilled into the humerus and the vise to secure the humerus (Figure 9). This prevented the bone from slipping from the vise due to the use of UFLs (>500 N). The free end of the wrapped tendon was placed in the upper grip of the test instrument. Tests and measurements are performed with a Test Resources 225LB Actuator (Shakopee, MN). A $10 \mathrm{~N}$ load is initially applied. The test force is then cycled 3500 times from 10 to $180 \mathrm{~N}$ at a rate of $635 \mathrm{~mm} / \mathrm{min}$ or until tendon failure. These parameters were chosen based on several biomechanical studies of human rotator cuff. ${ }^{3,17-19}$ Position and load were sampled at 100/s for every 20th cycle and later analysed using MATLAB (Ver 7.1)®. The initial $1 \mathrm{~min}$ of the test session was video recorded for measurement 
of gap formation using MaxTRAQ software (Ver2.2.2.5, Innovisionsystems.com). Photographs were taken at 100, 500, 1000, and 2000 cycles for gap measurement. Video recording at 30 frames/s of the single pull to failure test (UFL) was used to determine the mode of repair failure (STCamSWare x 86 ver1.0.0.4, www.sentechamerica. com). 10 intact tendons were subjected to the test sequence to ensure that this preparation was appropriate for the study. All survived the displacement-load cyclic test.

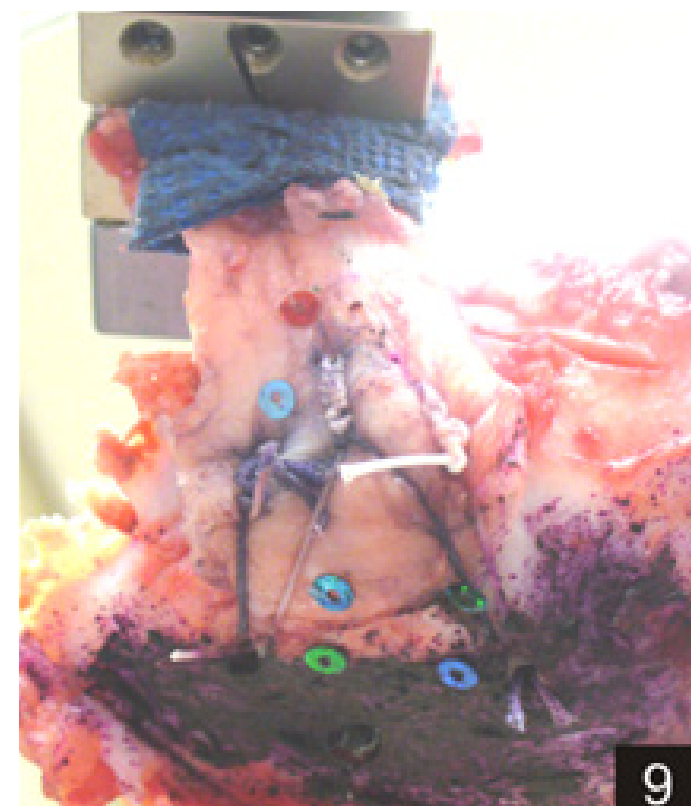

Figure 9 Illustration of the test apparatus with a NTR repaired tendon Multiple $3 \mathrm{~mm}$ markers are positioned on the tendon and bone that are used to determine gap size. Bone lateral to the end of the tendon was stained with Cresyl Violet to aid in gap determination.

\section{Results}

\section{Cyclic test}

The majority of repaired tendons survived the 3500 cycle test regardless repair type, this consisted of $84 \%$ of NTR and $61 \%$ of DR, and $64 \%$ of SR repairs. Gap size, a measure of repair integrity, is significantly larger for SR and DR compared to NTR (Figure 10). Gap size beyond 2000 cycles was nearly constant. NTR gap size is smaller than DR and SR gaps initially and over all later cycles of the test. With a commonly used criterion of a $5 \mathrm{~mm}$ gap constituting RCR failure, 29 of $39 \mathrm{DR}$ and 30 of 40 SR repairs failed compared to 7 of 37 NTR repairs. With a criterion of a $9 \mathrm{~mm}$ gap constituting repair failure then $14 \mathrm{DR}$ and $16 \mathrm{SR}$ repairs failed compared to zero NTR repairs. NTR repair gap size remained less than $3.1 \mathrm{~mm}$ in 14 instances throughout the 3500 test cycles. Because SR and DR results are very similar, NTR repair is only compared to the DR result. There is little overlap in gap size between techniques. An unpaired t-test for two groups with both unequal variances and counts was used to determine the significance of the gap differences ${ }^{20}$ At all three cycle counts the two-tailed p-value was less than 0.0001 . The t-value and degrees of freedom $(\mathrm{t}, \mathrm{df})$ for the three cycle counts were: at 100 cycles $(6.9,74)$, at 1000 cycles $(8.2,2)$ and at 2000 cycles $(8.3,53)$. All (t-values, dfs) indicate highly significant differences resulting in rejection of the $\mathrm{H} 0$ hypothesis based on gap size indicating an alternative hypothesis that NTR repair is a more robust technique. The mode of tendon failure was either intratendinous or intrasuture. $60 \%$ of SR and $64 \%$ of DR repairs failed at the sutures versus $33 \%$ of the NTR repairs. When an NTR RCR failed, the tendon tore above the sutures at twice the rate of the sutures pulling through the tendon.

\section{Ultimate failure load}

There was a significant difference between the mean UFLs for NTR versus SR repairs, $465 \mathrm{~N}$ versus $410 \mathrm{~N}$ (Table 1). The H0 hypothesis was checked with a t-test that yielded $\mathrm{t}=2.76$ with 44 degrees of freedom. The p-value for a one-sided test is 0.0042 and a 2 sided test of 0.0084 , both highly significant, resulting in the rejection of the H0 hypothesis. Based on UFL, NTR RCR is significantly better than either SR or DR repairs.

\section{Discussion}

The principal result of this study is that NTR RCR results in a smaller gap formation and higher UFLs than either SR or DR RCRs. This result was obtained while using higher loads $(180 \mathrm{~N})$ and significantly larger number of cycles (3500) than most previous studies. ${ }^{21,22}$ Based on this rigorous test environment it appears that after NTR repair the tendon will remain appropriately positioned at the footplate which is essential for healing after RCR.

There remains disagreement as what is the best RCR method. In an earlier study, SR repairs with a modified suture configuration were compared to four different DR repairs in pig shoulders with a sample size of $8 .^{21}$ Repairs were first tested with a 10 to $20 \mathrm{~N}$ force range for 50 cycles with the tendon at a $90^{\circ}$ angle to the humeral shaft. Maximum force was then increased in $20 \mathrm{~N}$ steps until a $100 \mathrm{~N}$ force was obtained. While DR repairs resulted in greater footprint coverage than SR, the principal finding was that SR repairs with modified suture configurations yielded comparable results to DR configurations. Similar results were obtained in this study with comparable gap sizes for SR and DR repairs (Figure 10) with the caveat is that SR repairs were more likely to fail based on excessive gap size formation.

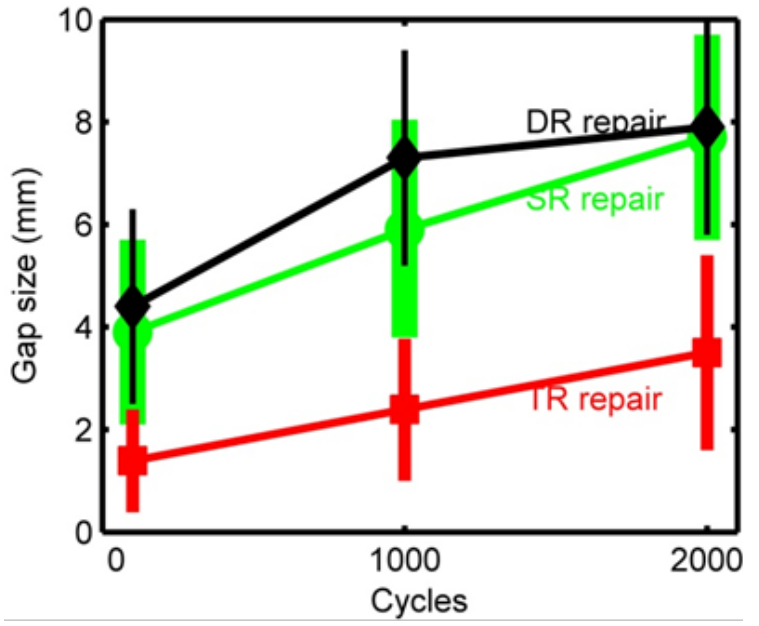

Figure 10 Comparison of mean gap formation for the three repair techniques at 100, 1000 and 2000 cycles. The vertical bars correspond to mean gap size in $\mathrm{mm} \pm$ standard deviation (SD).

A single row repair in cadavers utilized three triple loaded anchors to create a row of 6 mattress sutures and an additional two large mattress sutures close to the tendon edge. ${ }^{22}$ Cyclic loading results showed that this repair exhibited smaller displacements than a DR repair with significant differences between the anterior/posterior 
locations. In contrast, DR repairs were reported to be significantly better than SR repairs. ${ }^{23}$ The diamondback double row repair technique (DBK) resulted in a large "pressurized containment grid" in cadaver shoulders and used two double loaded anchors medially and three anchors laterally. ${ }^{14}$ DBK repair is similar to NTR repair in that both utilize lateral mattress sutures to maintain the lateral edge of the tendon in contact with the footplate. NTR repair has a double row of mattress stitches (total of four) and used one less anchor than DBK repair.

Table I UFLs statistics for the three repair techniques. Tendon failure during cyclic testing prevented UFL determination resulting in counts less than 40 , the group count for each technique

\begin{tabular}{llll}
\hline UFL & NTR & SR & DR \\
\hline Mean(N) & 465 & 414 & 394.5 \\
SD(N) & 88.8 & 77.1 & 65.4 \\
Count & 32 & 27 & 27 \\
$\operatorname{Min}(N)$ & 295 & 280 & 268 \\
$\operatorname{Max}(N)$ & 660 & 550 & 515
\end{tabular}

UFL, Ultimate Failure Load; results for each repair technique; Mean UFL, SD, Standard Deviation, Minimum, and Maximum are given in Newtons (N)

Anchor pullout and suture breakage have been studied extensively because of their importance in RCRs. ${ }^{24}$ Certain anchor design features may result in more eyelet and suture failures. For example, Barber demonstrated that lateral anchors that were designed to accommodate three sutures had significant eyelet failures and were more prone to suture slippage. ${ }^{25}$ A suture-tape, rip-stop DR configuration covered more of the footprint than a SR triple loaded anchor. ${ }^{26}$ No SR or DR repair tested showed $5 \mathrm{~mm}$ of displacement after the first 100 cycles. The most common catastrophic failure was the suture tearing out of the tendon. In this study, if a $5 \mathrm{~mm}$ gap is the criteria for repair failure then both SR and DR repairs failed as the number of cycles increased. In contrast, gap formation in NTR repairs never reached the $5 \mathrm{~mm}$ failure criteria.

The most common NTR repair failure was the tendon fatiguing followed by tearing above the sutures. Anchors did not pullout nor did sutures break or wear out at the eyelets. This indicates that generic PEEK anchors and UHMWPE sutures will not be limiting elements of RCRs. This reinforces the general conclusion that the size of the tear and condition of the tendon and bone are the limiting aspects of achieving a repaired rotator cuff. Patient age is a major factor affecting bone density resulting in longer healing times and contributing to the higher rate of $\mathrm{RC}$ repair failure. ${ }^{27}$ Tendon cellularity and vascularity are markedly diminished at age 70 , bone quality is also inferior, resulting from osteoporosis of the greater tuberosity, cystic degeneration, and irregularity of cortical margins, all of which may significantly complicate anchor fixation. ${ }^{28}$ Hardware required for RCR methods can vary considerably for medium to large tendon tares. One method used 3 anchors and 9 sutures in a SR repair with 8 mattress sutures while 6 anchors and 6 sutures were used for a DR repair with 4 mattress sutures. ${ }^{22}$ In comparison, NTR repair used 4 anchors and 4 sutures saving significant costs. When the humerus real estate in a RCR is limited a single anchor would be used laterally. Less hardware translates into shorter surgery time.

\section{Limitations}

This is a biomechanical study and as such its immediate application to the clinical experience is limited. This is a zero time measure and as such provides no information regarding tendon healing. Porcine humerus is likely harder than human humerus bone and that may explain why there were no anchor pullouts in these tests whereas in cadaver studies or patients the humerus may be compromised increasing the likelihood of anchor pullout.

\section{Conclusion}

The principal result of this study is that NTR RCR results in a smaller gap formation and higher UFLs than either SR or DR RCRs. Neither generic anchors nor UHMWPE sutures failed in the 120 RCRs performed in this study, i.e. no anchors pulled out or eyelets broke. For NTR RCRs tendons tore medial to the sutures and only occasionally at the sutures.

\section{Clinical relevance}

NTR rotator cuff repairs exhibited smaller gap development and higher UFLs than SR or DR repairs while providing compression of the tendon to the footplate. No additional time, effort, or materials are required relative to DR repairs. Generic anchor use did not degrade repairs while reducing costs. Whether these properties will result in improved outcomes in humans will have to be demonstrated in a clinical trial.

\section{References}

1. Lubowitz JL, Provencher MT, Poehling GG. Single-row versus double-row rotator cuff repair: the controversy continues. Arthroscopy. 2011;27(7):880-882.

2. Burkkart SS, Daiz Pagan JL, Wirth MA, et al. Cyclic loading of anchor-based rotator cuff repairs: Confirmation of the tension overload phenomenon and comparison of suture anchor fixation with transosseous fixation. Arthroscopy. 1997;13(2):720-724.

3. Mazzocca AD, Millett, PJ, Guanche SA, et al. Anthroscopic single-row versus double row suture anchor rotator cuff repair. Am J Sports Med. 2005;33(12):1861-1868.

4. Abbi G, Espinoza L, Odell T, et al. Evaluation of 5 knots and 2 suture materials for arthroscopic rotator cuff repair: Very strong sutures can slip. Arthroscopy. 2006;22(1):38-43.

5. Kim DH, Elattrache NS, Tibone JE, et al. Biomechanical comparison of a single-row versus double-row suture anchor technique for rotator cuff repair. Am J Sports Med. 2006;34(3):407-414.

6. Smith CD, Alexander S, Hill AM, et al. A biomechanical Comparison of Single and Double-row fixation in arthroscopic rotator cuff repair. J Bone Joint Surg. 2006;88(11):2425-2431.

7. Cheung EV, Safran MR. Arthroscopic rotator cuff repair-Traditional anchor techniques. Operative Techniques in Sports Med. 2012;20(3):213219.

8. Saridakis P, Jones G. Outcomes of single-row and double-row arthroscopic rotator cuff repair: a systematic review. J Bone Joint Surg Am. 2010;92(3):732-742.

9. Park JY, Lhee SH, Choi JH, et al. Comparison of the Clinical Outcomes of Single- and Double-row Repairs in Rotator Cuff Tears. Am J Sports Med. 2008;36(7):1310-1316 
10. Bicos J, Mazzocca AD, Hallab N, et al. The multi-suture technique for rotator cuff repair: A biomechanical evaluation. Orthopedics. 2007;30(11):910-919.

11. Jost PW, Khair M, Chen DX, et al. Suture number determines strength of rotator cuff repair. J Bone Joint Surg Am. 2012;94(14): e100.

12. Ostrander RV, McKinney BI. Evaluation of footprint contact area and pressure using a triple-row modification of the suture-bridge technique for rotator cuff repair. J Shoulder Elbow Surg. 2012;21(10):1406-1412.

13. Ostrander RV, Andrews J. Arthroscopic triple-row rotator cuff repair: A modified suture-bridge technique. Orthopedics. 2009;32(8):566-570.

14. Burkhart SS, Denard PJ, Obopilwe E, et al. Optimizing pressurized contact area in rotator cuff repair: the diamondback repair. Arthroscopy. 2012;28(2):188-195.

15. Burkhart SS, Cole JC. Bridging Self-Reinforcing Double-Row Rotator Cuff Repair: We Really Are Doing Better. Arthroscopy. 2010;26(5):677680 .

16. Clevenger TA, Beebe MJ, Strauss EJ, et al. The effect of insertion angle on the pullout strength of threaded suture anchors: A validation of the Deadman Theory. Arthroscopy. 2014;30(8):900-905.

17. Itoi E, Bergland LJ, Grabowski JJ, et al. Tensile properties of the supraspinatus tendon. J Ortho Res. 1995;13(4):578-584.

18. Burkhart SS, Diaz Pagan JL, Wirth MA, et al. Cyclic loading of anchor-based rotator cuff repairs: Confirmation of the tension overload phenomenon and comparison of suture anchor fixation with transosseous fixation. Arthroscopy. 1997;13(6):720-724.

19. Waltrip RL, Zheng N, Dugas JR, et al. Rotator cuff repair: a biomechanica comparison of three techniques. Am J Sports Med. 2003;31(4):493-497.
20. GraphPadSoftware.

21. Lorbach O, Bachelier F, Vees J, et al. Cyclic loading of rotator cuff reconstructions: single-row repair with modified suture configurations versus double-row repair. Am J Sports Med. 2008;36(8):1504-1510.

22. Lorbach O, Pape D, Raber F, et al. Influence of the initial rupture size and tendon subregion on the three-dimensional properties of single-row and double-row rotator cuff reconstructions. Knee Surg Sports Traumatol Arthrosc. 2012;20(11):2139-2147.

23. Ma B, Comerford L, Wilson J, et al. Biomechanical Evaluation of Arthroscopic Rotator Cuff Repairs: Double-Row Compared with SingleRow Fixation. J Bone Joint Surg. 2006;88(2):403-410.

24. Barber FA, Herbert MA, Hapa O, et al. Biomechanical analysis of pullout strengths of rotator cuff and glenoid anchors: 2011 update. Arthroscopy. 2011;27(7):895-905.

25. Barber FA, Hapa O, Bynum JA. Comparative testing by cyclic loading of rotator cuff suture anchors containing multiple high-strength sutures. Arthroscopy. 2010;26(9):S134-S141.

26. Barber FA, Drew ORA. A biomechanical comparison of tendon-bone interface motion and cyclic loading between single-row, triple-loaded cuff repairs and double-row, suture-tape cuff repairs using biocomposite anchors. Arthroscopy. 2012;8(9):1197-1205.

27. Nho SJ, Yadav H, Shindle MK, et al. Rotator Cuff Degeneration Etiology and Pathogenesis. Am J Sports Med. 2008;36(5):987-993.

28. Brewer BJ. Aging of the rotator cuff. Am J Sports Med. 1979;7(2):102110 . 